\title{
A halo blue straggler on a highly eccentric retrograde orbit ${ }^{\star} \star \star$
}

\author{
A. Tillich ${ }^{1}$, N. Przybilla ${ }^{1}$, R.-D. Scholz ${ }^{2}$, and U. Heber ${ }^{1}$ \\ 1 Dr. Remeis-Sternwarte Bamberg \& ECAP, Astronomisches Institut, Friedrich-Alexander-Universität Erlangen-Nürnberg, \\ Sternwartstr. 7, 96049 Bamberg, Germany \\ e-mail: Alfred.Tillich@sternwarte.uni-erlangen.de \\ 2 Astrophysikalisches Institut Potsdam, An der Sternwarte 16, 14482 Potsdam, Germany
}

Received 24 February 2010 / Accepted 2 April 2010

ABSTRACT

\begin{abstract}
Context. Blue stragglers, which are stars that appear to be younger than they should be, are an important population of unusual stars in both stellar clusters and the halo field of the Galaxy. Most formation scenarios evoke either stellar collisions or binary stars that transfer mass or merge.

Aims. We investigate high-velocity stars in the Galactic halo and perform a spectral and kinematical analysis to shed light on their nature and origin. Here we report that SDSSJ130005.62+042201.6 (J1300+0422 for short) is an A-type star of unusually large radial velocity $\left(504.6 \pm 5 \mathrm{~km} \mathrm{~s}^{-1}\right)$.

Methods. From a quantitative NLTE (and LTE) spectral analysis of medium-resolution optical spectra, the elemental composition is derived. Proper motion measurements combined with a spectroscopic distance estimate allow us to determine its present space velocity. Its kinematical properties are derived by integrating the equation of motion in the Galactic potential.

Results. We find $\mathrm{J} 1300+0422$ to be metal poor $([\mathrm{M} / \mathrm{H}]=-1.2)$ and exhibit an $\alpha$-element enrichment $(0.3-0.4$ dex $)$ that is characteristic of the halo population, as confirmed by a kinematical analysis of its 3D space motions, which places it on a highly eccentric retrograde Galactic orbit.

Conclusions. The mass of $\mathrm{J} 1300+0422\left(1.15 \pm 0.10 M_{\odot}\right)$ is higher than the globular cluster turn-off masses indicating that it is a halo blue straggler star. At a Galactic rest-frame velocity of $\approx 467 \mathrm{~km} \mathrm{~s}^{-1}$, the star travels faster than any known blue straggler but is still bound to the Galaxy.
\end{abstract}

Key words. stars: kinematics and dynamics - stars: individual: SDSSJ130005.62+042201.6 - blue stragglers - stars: Population II stars: atmospheres - Galaxy: halo

\section{Introduction}

Blue straggler stars (BSS) were first discovered as an unusual subclass of stars in globular clusters (Sandage 1953). They lie on or near the main sequence but are more luminous than the turn-off stars indicating that they are of higher mass than the latter. Apparently, BSS are present in all the Galactic globular clusters (Piotto 2003).

Because the stars in a cluster are believed all to have formed at the same time, whereas the stellar turnoff age decreases with mass, BSs should have evolved off the main sequence to become giants and white dwarfs long ago. It is generally believed that blue stragglers are coeval with the remaining stars in the cluster and originate in normal main-sequence stars that gained mass by means of a recent accretion episode. Most formation scenarios evoke mass transfer in and/or the merger of binary stars or collisions of stars.

It remains unclear whether two types of BSS exist in clusters, which may result from the different suggested mechanisms discussed frequently in the literature. The discovery of two distinct sequences of BSS in the globular cluster M 30 (Ferraro et al. 2009) indicates that indeed both formation mechanisms are at work.

\footnotetext{
* Based on data from the Sloan Digital Sky Survey.

$\star \star$ Based on observations at the $3.5 \mathrm{~m}$ telescope at DSAZ observatory (Calar Alto) in Spain. Program ID: H09-3.5-008.
}

In open clusters, this does not seem to be the case, because Mathieu \& Geller (2009) indentified a high frequency of binaries among the blue stragglers in the open cluster of NGC 188, most having eccentric orbits with periods of about 1000 days. Brown et al. (2009) argue that most if not all BSS in open clusters arise from multiple star systems. This suggests that blue stragglers are formed in both ways in star clusters, with collisions/mergers becoming more common with increasing cluster density.

In the Galactic field, it is more difficult to identify BSS because the stellar age cannot be determined. Field blue stragglers are therefore usually identified as metal-poor main sequence objects that are hotter than globular cluster main-sequence turnoff stars. Nevertheless, many blue metal-poor stars $[\mathrm{Fe} / \mathrm{H}]<-1$ with main-sequence luminosities have been found to be hotter than the main-sequence turnoff of globular clusters and are therefore considered to be field analogs of the cluster BSS population. These metal-poor stars seem to be so numerous that their specific frequency of appearance relative to regular horizontal branch stars was found to be higher than in globular clusters (Preston \& Sneden 2000). Since the stellar density is low in the field, binary star evolution and mass transfer is probably the most common path of formation among field BSS. Carney et al. (2001, 2005) pointed out that some BS candidates among metalpoor halo main-sequence stars are not binaries, hence binary star evolution and mass transfer may not be the only path. They argue that the apparently single stars could be the partial remnants of an accreted dwarf satellite galaxy whose star formation 
continued over a long period of time. These metal-poor mainsequence stars are simply therfore young stars.

A detailed spectral analysis of a blue metal-poor star sample in the field by Preston \& Sneden (2000) measured a very high binary fraction of at least $67 \%$, dominated by long period (wide) binaries. This population appears to be very similar to that of the open cluster NGC 188 (Mathieu \& Geller 2009). Assuming a formation by Roche lobe overflow during the red giant branch stage of the primary, Preston \& Sneden (2000) identified at least half of the blue metal-poor stars in their survey as blue stragglers. This is supported by more recent and much larger surveys for faint blue stars in the halo. For example, Xue et al. (2008) found that blue stragglers account for half of their sample of more than 10000 A-type stars.

We have embarked on a search for so-called hyper-velocity stars (HVS, Tillich et al. 2009) based on the work of Xue et al. (2008), who presented radial velocities for a large sample of blue stars. Their sample is a mix of blue horizontal branch (BHB), blue straggler, and main-sequence stars with effective temperatures roughly between 7000 and $10000 \mathrm{~K}$ according to their colours.

To study the stellar motions in $3 \mathrm{D}$, we need to derive their tangential velocities from proper motions and spectroscopic distances. The measurement of proper motions for faint highvelocity stars is the most challenging part of this project because the stars' distances are large and therefore require highly accurate proper motion measurements.

We focused on the fastest stars in terms of large Galactic rest-frame (GRF) velocities with the aim of determining their nature, distance, and kinematics from detailed quantitative spectral analyses and astrometry. First results have already been reported (Tillich et al. 2009; Przybilla et al. 2010). We found J0136+2425 to be an A-type main-sequence star travelling at $\approx 590 \mathrm{~km} \mathrm{~s}^{-1}$, possibly unbound to the Galaxy (Tillich et al. 2009), which makes it an excellent HVS candidate. More importantly, it was inferred to have originated in the outer Galactic rim, nowhere near the Galactic centre, which would be the favoured place of origin if a supermassive black hole acts as a slingshot as suggested by Hills (1988, see also Brown et al. 2009). Amongst the stars of negative GRF velocity, we discovered that J1539+0239 is a BHB travelling with the largest space velocity of any BHB star known so far, which allowed us to place a lower limit on the mass of the Galactic halo (Przybilla et al. 2010).

Here we report that SDSSJ130005.62+042201.6 (J1300+0422 for short) is a metal-poor blue straggler of Population II on a wide retrograde halo orbit that is only marginally bound to the Galaxy in a standard Galactic potential.

\section{Target selection and proper motions}

We selected all stars with GRF velocities $v_{\mathrm{GRF}}>+350 \mathrm{~km} \mathrm{~s}^{-1}$ from the RV-based sample of Xue et al. (2008) and obtaining 11 targets for which we attempted to measure proper motions (Tillich et al. 2009). All available independent position measurements from Schmidt plates (APM - McMahon et al. 2000; SSS - Hambly et al. 2001) were collected and combined with the SDSS and other available positions (CMC14 CarlsbergMeridian-Catalog 2006; 2MASS - Cutri et al. 2003; UKIDSS - Lawrence et al. 2007) to perform a first linear proper-motion fit. However, there were even more measurements from Schmidt plates, for up to 14 different epochs in the case of overlapping plates of the Digitised Sky Surveys ${ }^{1}$. FITS images of 15 by

\footnotetext{
${ }^{1}$ http://archive.stsci.edu/cgi-bin/dss_plate_finder
}
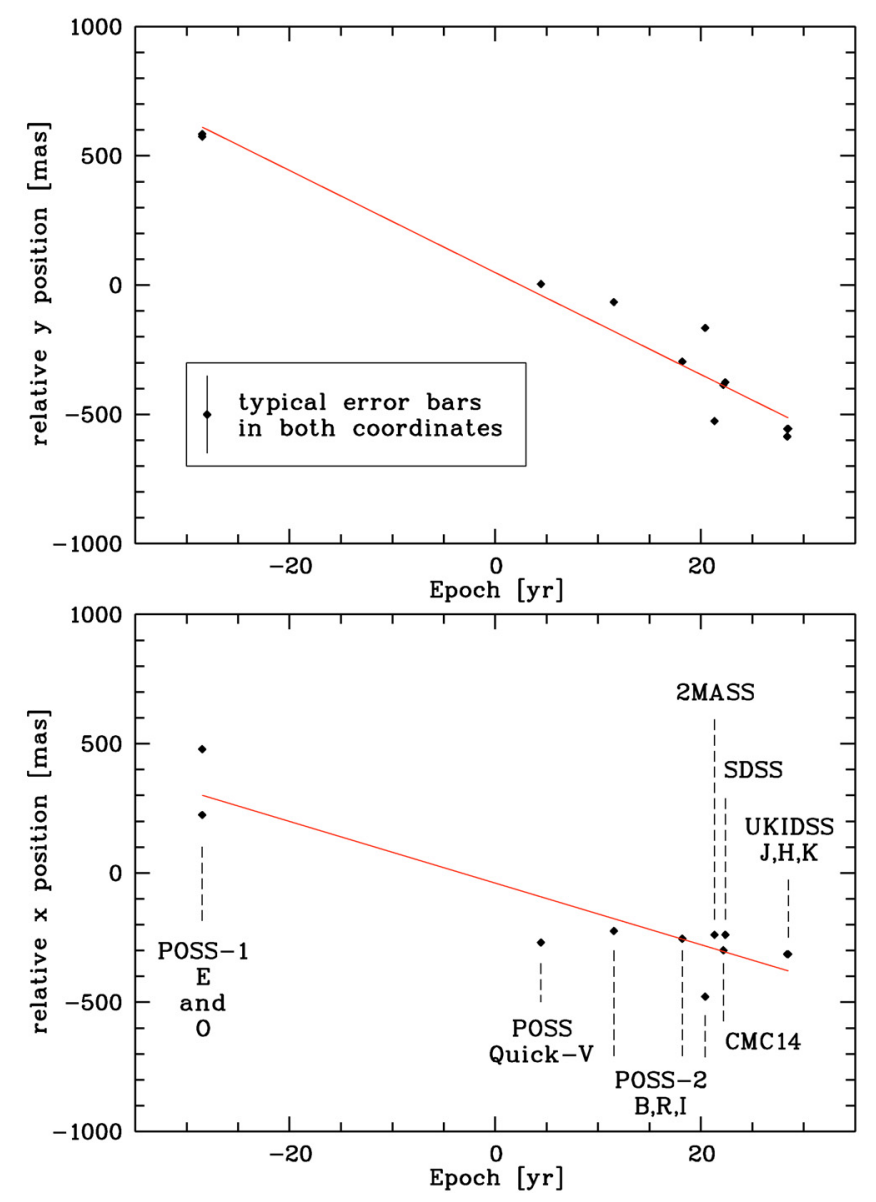

Fig. 1. Linear fit of the position measurements for J1300+0422, where 1978.84 is the zero epoch.

15 arcmin size were extracted from all available plates and ESO MIDAS tools were used to measure positions. For this purpose, we selected compact background galaxies around each target, identified from $S D S S$, to transform the target positions on all the Schmidt plates to the SDSS system. The small fields allowed us to apply a simple model (shift+rotation) and to achieve a higher accuracy in our final proper motion fit for all our targets (see e.g. Figs. 1 and A.1). Proper motions differing significantly from zero were found for the three brightest stars only ${ }^{2}$. The results for $\mathrm{J} 1300+0422$ are given in Table 1 .

\section{Observations and quantitative spectroscopy}

To search for radial velocity variations, we reobserved J1300+0422 with the TWIN spectrograph at the 3.5m telescope on Calar Alto on May 2009. The radial velocity (heliocentric $v_{\text {rad }}=515.3 \pm 8 \mathrm{~km} \mathrm{~s}^{-1}$ ) was derived by $\chi^{2}$-fitting of appropriate synthetic spectra over the full spectral range ${ }^{3}$. It was found to be consistent with that measured from the SDSS spectrum (heliocentric $v_{\mathrm{rad}}=504.6 \pm 5 \mathrm{~km} \mathrm{~s}^{-1}$ ).

2 SDSSJ155352.41+003012.0 (J1553+0030 for short) turned out to be a spectroscopic binary and will not be studied further in this paper. For the sake of completeness, we provide details in the Appendix

${ }^{3}$ Because we used many spectral lines our results differ from those of Xue et al. (2008), who used cross-correlation for RV and then the $\mathrm{H} \gamma+\mathrm{H} \delta$ lines for stellar-type determination only. 
A. Tillich et al.: A halo blue straggler on a highly eccentric retrograde orbit

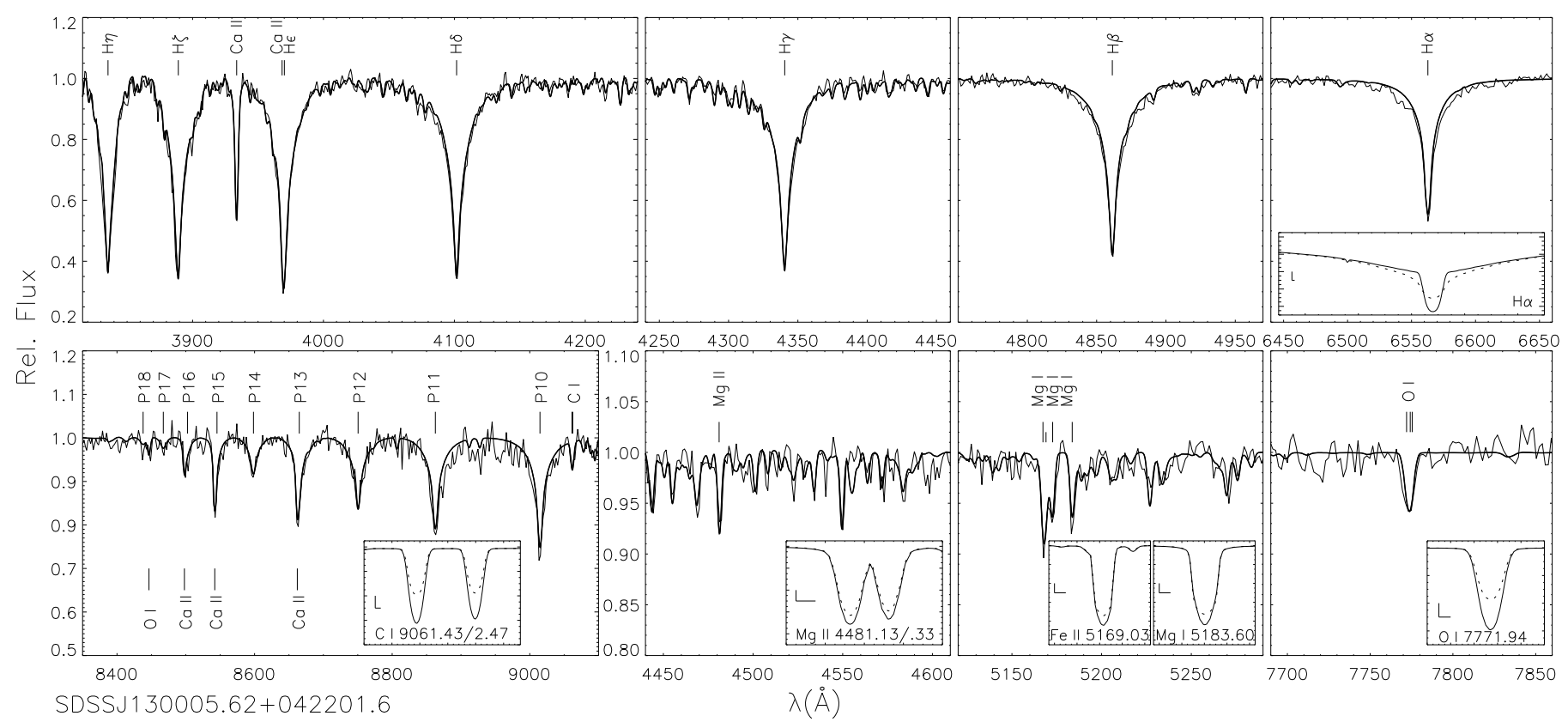

Fig. 2. Comparison of NLTE spectrum synthesis (thick line) with observation (thin wiggly line) for J1300+0422. Displayed are selected regions around the Balmer lines, the higher Paschen series, Mg II $\lambda 4481 \AA$, the Mg I b, and the near-IR O I triplets. The insets compare computed NLTE (full) and LTE profiles (dotted lines) for several key features, as indicated. No convolution by instrumental or rotational profiles was applied to exemplify what can be expected at high spectral resolution. The markers are extended horizontally by $0.1 \AA$ and vertically by 0.1 units.

Table 1. Results of the analysis of $\mathrm{J} 1300+0422$.

\begin{tabular}{lc|lc}
\hline \hline$V(\mathrm{mag})^{a}$ & $15.12 \pm 0.02$ & $E(B-V)(\mathrm{mag})^{b}$ & $0.05 \pm 0.02$ \\
$\mu_{\alpha} \cos (\delta)\left(\mathrm{mas} \mathrm{yr}^{-1}\right)$ & $-11.9 \pm 1.7$ & $\mu_{\delta}\left(\mathrm{mas} \mathrm{yr}^{-1}\right)$ & $-19.7 \pm 1.4$ \\
$n_{\mathrm{ep}}$ & 12 & $n_{\mathrm{gal}}$ & 15 \\
$T_{\mathrm{eff}}(\mathrm{K})$ & $7350 \pm 200$ & $\log g(\mathrm{cgs})$ & $4.00 \pm 0.15$ \\
{$[M / H]$} & $-1.2 \alpha$ & $M / M_{\odot}$ & $1.15 \pm 0.10$ \\
$v_{\mathrm{rad}}\left(\mathrm{km} \mathrm{s}^{-1}\right)$ & $504.6 \pm 5$ & $v_{\mathrm{rot}} \sin i\left(\mathrm{~km} \mathrm{~s}^{-1}\right)$ & - \\
$d\left(\mathrm{kpc}^{-1}\right.$ & $3.25 \pm 0.62$ & $v_{\mathrm{GRF}}\left(\mathrm{km} \mathrm{s}^{-1}\right)$ & $467_{-21}^{+41}$ \\
$v_{\mathrm{esc}}\left(\mathrm{km} \mathrm{s}^{-1}\right)$ & 529 & $v_{\mathrm{esc}}^{\mathrm{a}}$ & 708 \\
\hline
\end{tabular}

Notes. The resulting GRF velocity $v_{\mathrm{GRF}}$ and the local escape velocity $v_{\text {esc }}$, also in a more massive halo $v_{\text {esc }}^{\mathrm{a}}$, are given. ${ }^{(a)}$ The visual magnitude has been derived from SDSS photometry following Jordi et al. (2006). (b) The interstellar colour excess $E(B-V)$ has been determined by comparing the observed colours to synthetic ones from the model spectral energy distribution.

We also inspected three individual SDSS spectra that were acquired one after the other. No variations were found. This indicates that large radial velocity variations on small timescales are unlikely, but we cannot rule out small RV variations over long periods as observed in many BSS (Carney et al. 2005).

A quantitative analysis of the coadded SDSS spectrum of J1300+0422 was carried out following the hybrid NLTE approach discussed by Przybilla et al. (2006). In brief, line-blanketed LTE model atmospheres were computed with ATLAS9 (Kurucz 1993) and NLTE (and LTE) line-formation calculations were performed using updated versions of DETAIL and Surface (Giddings 1981; Butler \& Giddings 1985). Many astrophysical important chemical species were treated in NLTE, using state-of-the-art model atoms (H: Przybilla \& Butler 2004a; C I: Przybilla et al. 2001b; N I: Przybilla \& Butler 2001; O I: Przybilla et al. 2000; Mg I/II: Przybilla et al. 2001a; Ti II and Fe II: Becker 1998).
The effective temperature $T_{\text {eff }}$ and the surface gravity $\log g$ were determined by fits to the Stark-broadened Balmer and Paschen lines and the ionisation equilibrium of $\mathrm{Mg} \mathrm{I} / \mathrm{II}$. We adopted a microturbulent velocity of $2 \mathrm{~km} \mathrm{~s}^{-1}$ and a mixinglength to pressure-scale-height ratio $\ell / H$ of 1.25 for the convective atmosphere. The stellar metallicity was derived by model fits to the observed metal-line spectra. Results are listed in Table 1 and a comparison of the resulting final synthetic spectrum with observations is shown in Fig. 2. Overall, excellent agreement is obtained for the strategic spectral lines throughout the entire wavelength range. The uncertainties in the stellar parameters were constrained by the quality of the match of the spectral indicators within the given $\mathrm{S} / \mathrm{N}$ limitations.

It may be instructive at this point to take a closer look at the spectrum synthesis in both NLTE and LTE, as this has not been done so far for Population II blue straggler stars ${ }^{4}$. A few comparisons of NLTE and LTE profiles are therefore shown in the insets in Fig. 2. The combination of a higher $T_{\text {eff }}$ than typically found for Population II stars and the diminished line blocking because of the low metal content results in a hardened radiation field that caused pronounced NLTE effects on many diagnostic lines. NLTE strengthening is found for the Doppler core of $\mathrm{H} \alpha$ - in line with the behaviour in cooler (e.g. Przybilla \& Butler 2004b) as well as in hotter stars (e.g. Nieva \& Przybilla 2007) but the inner line wings are weakened. The higher Balmer and Paschen lines show much lower deviations from LTE. Our calculations predict that the majority of the weak metal-lines are described well by the assumption of LTE. On the other hand, many of the stronger metal lines, such as e.g., those shown in the insets in Fig. 2, exhibit pronounced NLTE strengthening. To reproduce the NLTE equivalent widths of these particular lines, we need to

\footnotetext{
${ }^{4}$ De Marco et al. (2005) presented global fits of partially lineblanketed NLTE model atmospheres to several blue straggler stars in globular clusters. However, the differences between NLTE and LTE model spectra were not elaborated on there.
} 


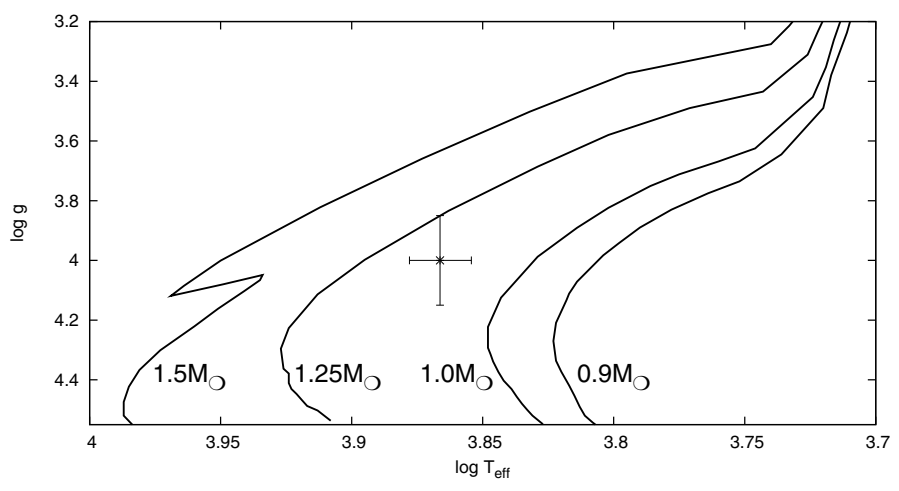

Fig. 3. Comparison of the position of $\mathrm{J} 1300+0422$ in the $\left(T_{\text {eff }}, \log g\right)$ diagram to evolutionary tracks of Schaller et al. (1992) for subsolar metallicity $(Z=0.001)$, to determine its mass.

apply abundance corrections of about $+0.2 \mathrm{dex}(\mathrm{Mg} \mathrm{I} / \mathrm{II}, \mathrm{Fe}$ II $)$, $+0.5 \mathrm{dex}(\mathrm{O} \mathrm{I})$, and $+0.8 \mathrm{dex}(\mathrm{C} \mathrm{I})$ in LTE. We note, however, that these line are close to being saturated. LTE computations for higher abundances cannot therefore not reproduce the NLTE line depths at all, but instead have stronger line wings. Abundance studies based on equivalent widths may therefore be misleading, because these differences remain unnoticed. An investigation at high spectral resolution would therefore be worthwhile to facilitate the NLTE effects to be studied in detail.

Its measurement of $T_{\text {eff }}$ and gravity places $\mathrm{J} 1300+0422$ on the main sequence (see Fig. 3) at a mass of $1.15 M_{\odot}$ as derived by comparing the position of the star to predictions of the evolutionary models of Schaller et al. (1992). No rotational broadening was detected. The metallicity is lower than solar by a factor of almost 20 and the abundances of the $\alpha$-elements (those from $\mathrm{O}$ to $\mathrm{Ti}$ ) are enhanced by $\sim 0.3-0.4 \mathrm{dex}$, which is typical of the halo population. This suggests that star is a halo blue straggler. All results are summarised in Table 1.

\section{Distance, kinematics, and errors}

Using the mass, effective temperature, gravity, and extinctioncorrected apparent magnitude we derive the distance following Ramspeck et al. (2001) using the fluxes from the final model spectrum. The distance error is dominated by the gravity error.

Applying the Galactic potential of Allen \& Santillan (1991), we calculated orbits and reconstructed the path of the star through the Galactic halo with the program of Odenkirchen \& Brosche (1992). The distance of the GC from the Sun was adopted to be $8.0 \mathrm{kpc}$ and the Sun's motion with respect to the local standard of rest was taken from Dehnen \& Binney (1998). Since the RV is well known, the error in the space motion is dominated by that of the distance, which is affected most by the gravity error, and those of the proper motion components. Varying these three quantities within their respective errors, we applied a Monte Carlo procedure to derive the distribution of GRF velocities at the present location and its median (see Fig. 4). The local escape velocity was calculated by assuming the Galactic potential of Allen \& Santillan (1991). The GRF velocity of $467_{-21}^{+41} \mathrm{~km} \mathrm{~s}^{-1}$ therefore remains below the local escape velocity of $v_{\mathrm{esc}} \approx 529 \mathrm{~km} \mathrm{~s}^{-1}$ and $\mathrm{J} 1300+0422$ is bound to the Galaxy.

To quantify the kinematics of the star more accurately, we compared our results with those of Pauli et al. (2006). Based on the 3D-orbit, the $V-U$ diagram, the eccentricity $e$, and the the $Z$-component of the angular momentum $J_{Z}$ these authors introduced a kinematic population classification scheme

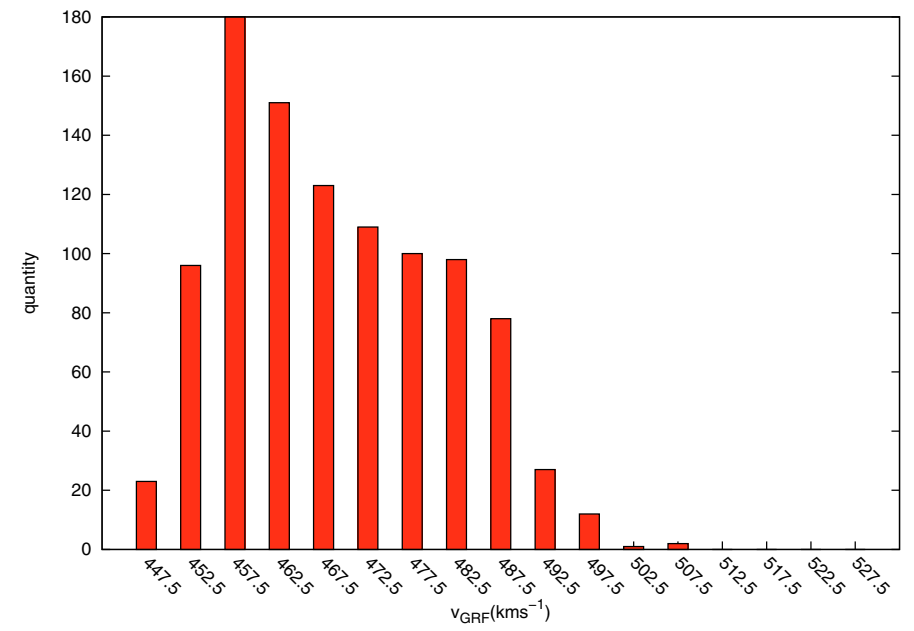

Fig. 4. Galactic rest-frame velocity distribution for J1300+0422 derived from a Monte Carlo procedure with a depth of 1000.

and combined it with age information. They analysed 398 DA white dwarfs from the SPY project, the largest homogeneous sample of its kind, and performed a detailed kinematic analysis accounting for errors using a Monte Carlo error propagation code, as in our method. A substantial thick disk fraction of $7 \%$ was found, while $2 \%$ of the DAs appeared to have characteristic halo properties.

Following this approach, we found that J1300+0422 clearly belongs to the halo population. This is already obvious in Fig. 5, which shows the characteristic $V-U$ and $-e-J_{Z}$ diagrams in relation to the reference white dwarf sample of Pauli et al. (2006). We note that the orbit of $\mathrm{J} 1300+0422$ is retrograde and highly eccentric. Furthermore, the star travels far out into the halo out to distances of more than $100 \mathrm{kpc}$ (see Fig. 6). Whether the star is bound to the Galaxy depends on the Galactic potential adopted, in particular on the dark matter halo, as pointed out by Abadi et al. (2009). In our standard potential (Allen \& Santillan 1991), we adopted a halo mass out to $100 \mathrm{kpc}$ of $M_{\text {Halo }}=8 \times 10^{11} M_{\odot}$. The analysis of J1539+0239 (Przybilla et al. 2010) inferred a higher halo mass of at least $M_{\text {halo }} \sim 1.7_{-1.1}^{+2.3} \times 10^{12} M_{\odot}$. We therefore repeated our analysis accordingly and found a higher escape velocity of $v_{\mathrm{esc}} \approx 708 \mathrm{~km} \mathrm{~s}^{-1}$, which means that the star would in any case be bound. The effect on the angular momentum was negligible, while the orbit became significantly less eccentric (see Fig. 5). Hence the star might have crossed the disk even more often within its lifetime.

\section{Conclusion}

We have presented a quantitative spectral analysis of a highvelocity star from the sample of faint blue stars in the halo of Xue et al. (2008). Its radial velocity, proper motion, and spectroscopic distance were derived and a detailed kinematical analysis was performed using the Galactic potential of Allen \& Santillan (1991) as well as a potential modified one that assumes a more massive dark matter halo.

The metal-poor A-type star J1300+0422 was identified as a blue straggler of $1.15 M_{\odot}$ due to its main-sequence gravity. A detailed NLTE analysis was performed, which we compared to the standard LTE approach. Significant differences were found especially for $\mathrm{C}$ I and $\mathrm{O}$ I. With its low metallicity of $[\mathrm{Fe} / \mathrm{H}]=-1.2$ and characteristic enhancement of $\alpha$-elements, it would fit perfectly into the sample of Preston \& Sneden (2000), apart from the huge space velocity of the star. The kinematic characteristics 
A. Tillich et al.: A halo blue straggler on a highly eccentric retrograde orbit
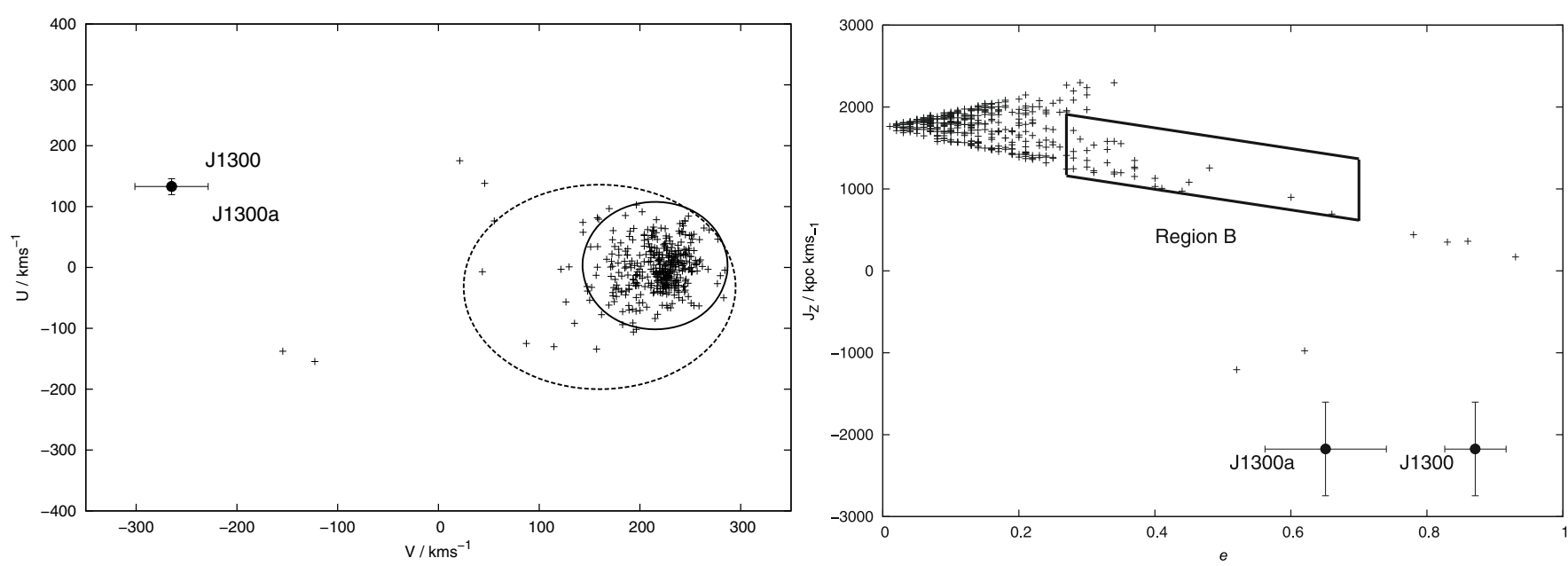

Fig. 5. $V-U$ (left) and $e-J_{Z}$ diagram (right) for J1300+0422. In case of a higher Galactic halo mass (Przybilla et al. 2010) the position is marked with J1300a. The white dwarf sample (+) of Pauli et al. (2006) serves as reference. The ellipses in the $V-U$ diagram indicate the thin and thick disk contours, halo stars lie outside of these ellipses. The left-hand triangle-shaped cloud of stars are the thin disk white dwarf population, while the solid box marks the thick disk region (Region B). Note that in both diagrams J1300+0422 lies far away from the disk populations.

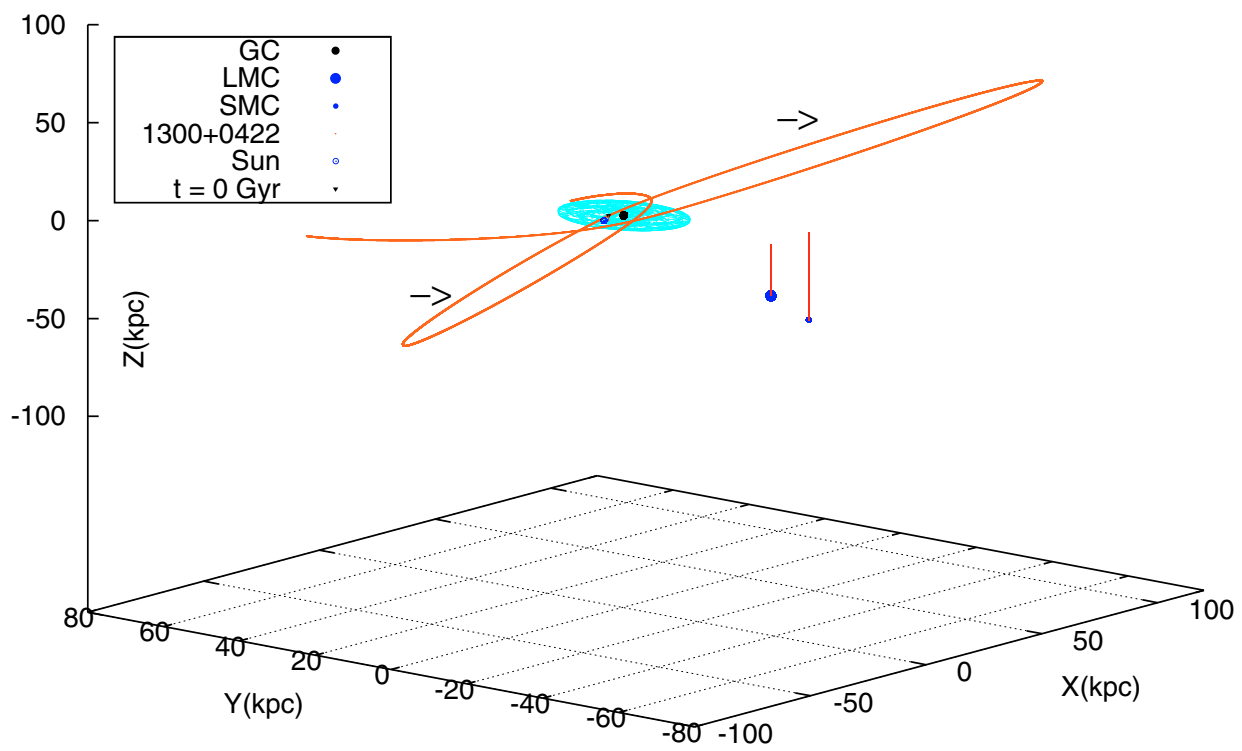

Fig. 6. Trajectory of the metal-poor blue straggler J1300+0422, relative to the Galactic disk (grey), calculated for \pm 2 Gyrs in time. The current position is marked.

$\left(U, V, e, J_{\mathrm{Z}}\right.$ ) confirm the halo membership of $\mathrm{J} 1300+0422$ beyond any doubt. In addition, its trajectory continues far out into the halo. Many blue stragglers were found to be long period binaries (with periods of several 100 to 1000 days; Carney et al. 2005; Mathieu \& Geller 2009) with low radial velocity semi-amplitudes ( $K \sim 5-10 \mathrm{~km} \mathrm{~s}^{-1}$, Carney et al. 2001, 2005). Whether or not $\mathrm{J} 1300+0422$ is such a binary needs to be verified by an extensive radial velocity study.

Our kinematical result is limited by the errors in both the spectroscopic distance and the proper motion. ESA's upcoming astrometry mission GAIA will improve the situation because it will provide a parallax measurement with which to check the spectroscopic distance and improve the proper motion of J1300. GAIA will also have an enormous impact on research into blue stragglers in a more general sense as it will provide astrometry of thousands of halo BSS.

Acknowledgements. A.T. acknowledges funding by the Deutsche Forschungsgemeinschaft through grant HE1356/45-1. Travel to the Deutsch Spanisches
Astronomisches Zentrum (DSAZ, Calar Alto, Spain) was funded by DFG through grant He1356/50-1. We are very grateful to Stephan Geier for stimulating discussions and advice. Our thanks go to Sebstian Müller for observing and reducing the data from DSAZ. Funding for the SDSSand SDSS-II has been provided by the Alfred P. Sloan Foundation, the Participating Institutions, the National Science Foundation, the US Department of Energy, the National Aeronautics and Space Administration, the Japanese Monbukagakusho, the Max Planck Society, and the Higher Education Funding Council for England. The SDSSWeb Site is http://www. sdss.org/.

\section{Appendix A: J1553}

In the spectrum of $\mathrm{J} 1553+0030$, the Ca II IR triplet and to a lesser ecxtent the $\mathrm{Mg} \mathrm{I} b$ triplet are unusually strong, and most likely produced by a cool companion contributing to the spectra, hence we had to exclude $\mathrm{J} 1553+0030$ from the rest of our analysis. To confirm this result, we reobserved J1553+0030 with the TWIN spectrograph at the $3.5 \mathrm{~m}$ telescope on Calar Alto in May 2009. 
A\&A 517, A36 (2010)
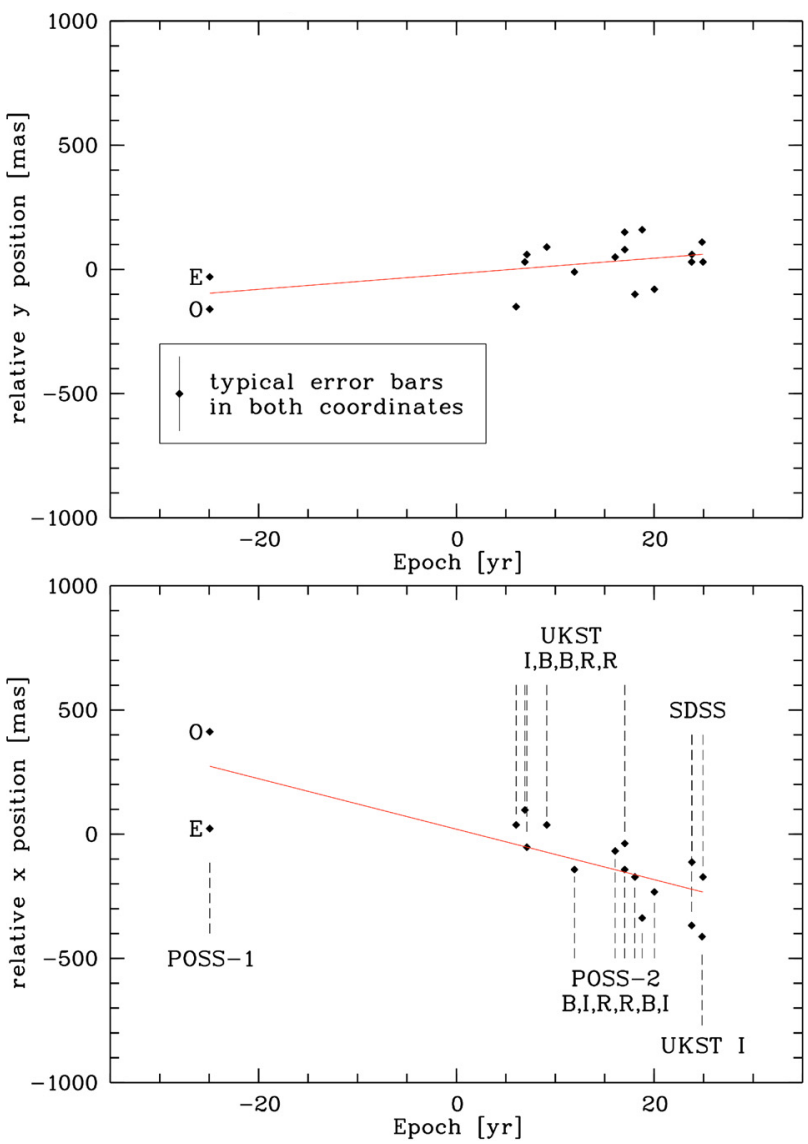

Fig. A.1. Linear fit to the position measurement for J1553+0030, where 1975.40 is the zero epoch. The PM was derived to be $\mu_{\alpha} \cos (\delta)=$

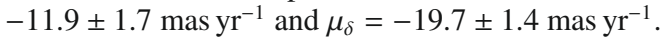

\section{References}

Abadi, M. G., Navarro, J. F., \& Steinmetz, M. 2009, ApJ, 691, L63 Allen, C., \& Santillan, A. 1991, Rev. Mex. Astr. Astrofis., 22, 255

Becker, S. R. 1998, in Properties of Hot Luminous Stars, ed. I. Howarth, ASP Conf. Ser., 131, 137
Brown, W. R., Geller, M. J., \& Kenyon, S. J. 2009, ApJ, 690, 1639

Butler, K., \& Giddings, J. R. 1985, Newsletter of Analysis of Astronomical Spectra, Univ. London, 9

Carlsberg-Meridian-Catalog. 2006, Copenhagen Univ. Obs., Inst. of Astr., Cambridge, UK, Real Inst. y Obs. de la Armada en San Fernando, 1304, 0

Carney, B. W., Latham, D. W., Laird, J. B., Grant, C. E., \& Morse, J. A. 2001, AJ, 122, 3419

Carney, B. W., Aguilar, L. A., Latham, D. W., \& Laird, J. B. 2005, AJ, 129, 1886 Cutri, R. M., Skrutskie, M. F., van Dyk, S., et al. 2003, 2MASS All Sky Catalog of point sources.

De Marco, O., Shara, M. M., Zurek, D., et al. 2005, ApJ, 632, 894

Dehnen, W., \& Binney, J. J. 1998, MNRAS, 298, 387

Ferraro, F. R., Beccari, G., Dalessandro, E., et al. 2009, Nature, 462, 1028

Giddings, J. R. 1981, PhD Thesis, , University of London

Hambly, N. C., MacGillivray, H. T., Read, M. A., et al. 2001, MNRAS, 326, 1279

Hills, J. G. 1988, Nature, 331, 687

Jordi, K., Grebel, E. K., \& Ammon, K. 2006, A\&A, 460, 339

Kurucz, R. 1993, ATLAS9 Stellar Atmosphere Programs CD-ROM No. 13. Smithsonian Astrophysical Observatory

Lawrence, A., Warren, S. J., Almaini, O., et al. 2007, MNRAS, 379, 1599

Mathieu, R. D., \& Geller, A. M. 2009, Nature, 462, 1032

McMahon, R. G., Irwin, M. J., \& Maddox, S. J. 2000, VizieR Online Data Catalog, 1267, 0

Nieva, M. F., \& Przybilla, N. 2007, A\&A, 467, 295

Odenkirchen, M., \& Brosche, P. 1992, Astron. Nachr., 313, 69

Pauli, E., Napiwotzki, R., Heber, U., Altmann, M., \& Odenkirchen, M. 2006, A\&A, 447, 173

Piotto, G. 2003, in New Horizons in Globular Cluster Astronomy, ed. G. Piotto, G. Meylan, S. G. Djorgovski, \& M. Riello, ASP Conf. Ser., 296, 263

Preston, G. W., \& Sneden, C. 2000, AJ, 120, 1014

Przybilla, N., \& Butler, K. 2001, A\&A, 379, 955

Przybilla, N., \& Butler, K. 2004a, ApJ, 609, 1181

Przybilla, N., \& Butler, K. 2004b, ApJ, 610, L61

Przybilla, N., Butler, K., Becker, S. R., Kudritzki, R. P., \& Venn, K. A. 2000, A\&A, 359, 1085

Przybilla, N., Butler, K., Becker, S. R., \& Kudritzki, R. P. 2001a, A\&A, 369, 1009

Przybilla, N., Butler, K., \& Kudritzki, R. P. 2001b, A\&A, 379, 936

Przybilla, N., Butler, K., Becker, S. R., \& Kudritzki, R. P. 2006, A\&A, 445, 1099 Przybilla, N., Tillich, A., Heber, U. \& Scholz, R.-D. 2010, ApJ, 718, 37

Ramspeck, M., Heber, U., \& Moehler, S. 2001, A\&A, 378, 907

Sandage, A. R. 1953, AJ, 58, 61

Schaller, G., Schaerer, D., Meynet, G., \& Maeder, A. 1992, A\&AS, 96, 269

Tillich, A., Przybilla, N., Scholz, R., \& Heber, U. 2009, A\&A, 507, L37

Xue, X. X., Rix, H. W., Zhao, G., et al. 2008, ApJ, 684, 1143 